livraisons

d'Histoire

de l'Architecture

\section{Livraisons de l'histoire de l'architecture}

$25 \mid 2013$

Architectures médiévales : types, matières et formes

\title{
Saint-Martin de Tours. Nouvelles propositions pour la datation du chevet du $\mathrm{XI}^{\mathrm{e}}$ siècle
}

Saint-Martin de Tours. New proposals for dating the $11^{\text {th }}$ century apse Die Stiftskirche Saint-Martin de Tours. Neue Vorschläge für die Datierung des Chorhauptes aus dem 11. Jahrundert

\section{Pierre Martin}

\section{(2) OpenEdition}

\section{Journals}

\section{Édition électronique}

URL : http://journals.openedition.org/lha/308

DOI : $10.4000 /$ /ha.308

ISSN : 1960-5994

\section{Éditeur}

Association Livraisons d'histoire de l'architecture - LHA

\section{Édition imprimée}

Date de publication : 10 juin 2013

Pagination : 83-96

ISSN : 1627-4970

\section{Référence électronique}

Pierre Martin, « Saint-Martin de Tours. Nouvelles propositions pour la datation du chevet du XIe siècle », Livraisons de l'histoire de l'architecture [En ligne], 25 | 2013, mis en ligne le 10 juin 2015, consulté le 19 avril 2019. URL : http://journals.openedition.org//ha/308 ; DOl : 10.4000//ha.308

Ce document a été généré automatiquement le 19 avril 2019

Tous droits réservés à l'Association LHA 


\title{
Saint-Martin de Tours. Nouvelles propositions pour la datation $\mathrm{du}$ chevet du XI ${ }^{\mathrm{e}}$ siècle
}

\author{
Saint-Martin de Tours. New proposals for dating the $11^{\text {th }}$ century apse \\ Die Stiftskirche Saint-Martin de Tours. Neue Vorschläge für die Datierung des \\ Chorhauptes aus dem 11. Jahrundert
}

\author{
Pierre Martin
}

1 Presque totalement détruite au lendemain de la Révolution française, la collégiale de Saint-Martin de Tours constitue néanmoins un jalon déterminant dans l'historiographie de l'architecture romane. Flanquée de deux tours, la façade était suivie par une longue nef de onze travées à cinq vaisseaux. Le vaste transept était doté de collatéraux et d'une tour-porche sur chacun de ses bras. Enfin, le chevet comportait un déambulatoire à cinq chapelles rayonnantes. Ce dernier fut reconstruit au début XIII ${ }^{e}$ siècle, après une transformation de la nef dans le dernier quart du XII ${ }^{e}$ siècle, mais les vestiges antérieurs furent reconnus en 1886, lors d'une campagne de fouilles préalables à la construction de la basilique moderne érigée sur les plans de Victor Laloux ${ }^{1}$.

De ces quelques éléments, il ressort de prime abord que le parti architectural de SaintMartin de Tours relevait des églises dites de pèlerinage, théorie établie au début des années 1920 par Émile Mâle². Outre les caractéristiques de son plan, l'église comptait, d'après l'iconographie ancienne, deux niveaux d'élévation - grandes arcades et tribunes considérés comme caractéristiques de ce groupe constitué également par Saint-Sernin de Toulouse, Saint-Martial de Limoges, Sainte-Foy de Conques, Saint-Jacques de Compostelle ${ }^{3}$ et, à l'origine, Saint-Remi de Reims, Saint-Sauveur de Figeac ou Saint-Gaudens.

3 S'il ne s'agit pas ici de résoudre les nombreux problèmes archéologiques posés par ce monument emblématique, on s'attachera à la datation du célèbre chevet de Saint-Martin, souvent reconnu comme un véritable modèle architectural. Les fondations qui furent mises au jour en 1886 répondaient à un hémicycle, précédé d'une travée droite et ceinturé d'un déambulatoire ouvrant sur une série de cinq chapelles rayonnantes. 
Tours (37), Saint-Martin, coupe longitudinale et plan des fouilles

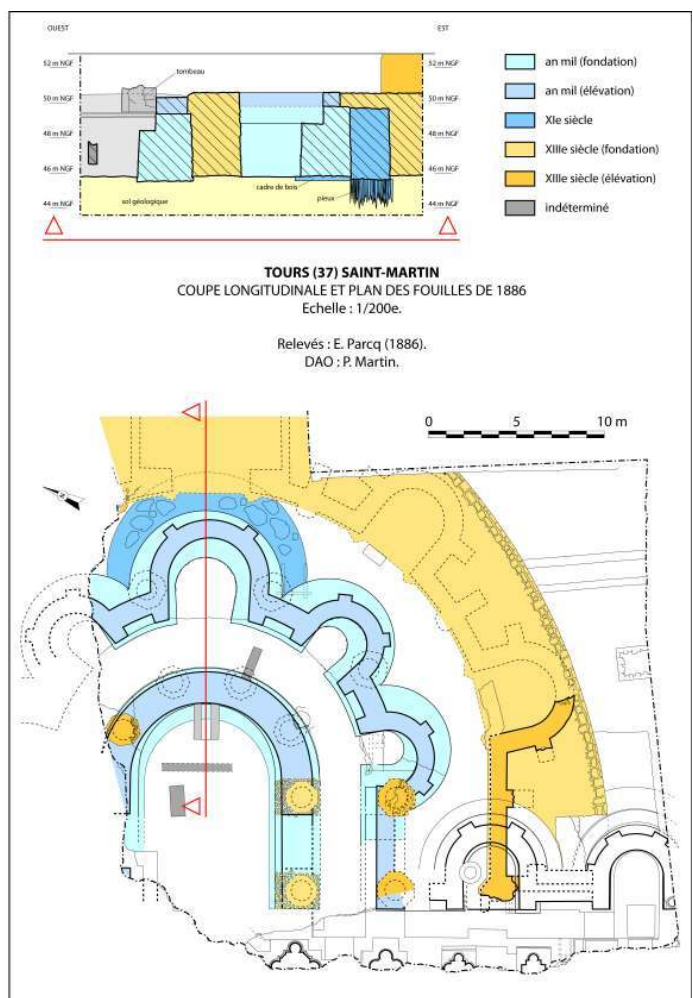

(1886). Échelle : 1/200

Relevés : E. Parcq. DAO : P. Martin

Des élévations, très arasées, il ne subsistait seulement qu'un petit muret semi-circulaire, qui délimitait l'abside abritant le tombeau de saint Martin, et le déambulatoire lisible de l'extérieur. Il n'en reste aujourd'hui plus que les fondations de ce dernier et une infime partie de l'élévation de la chapelle rayonnante axiale, accessibles depuis la crypte de la basilique moderne. 
Tours (37), basilique Saint-Martin, crypte

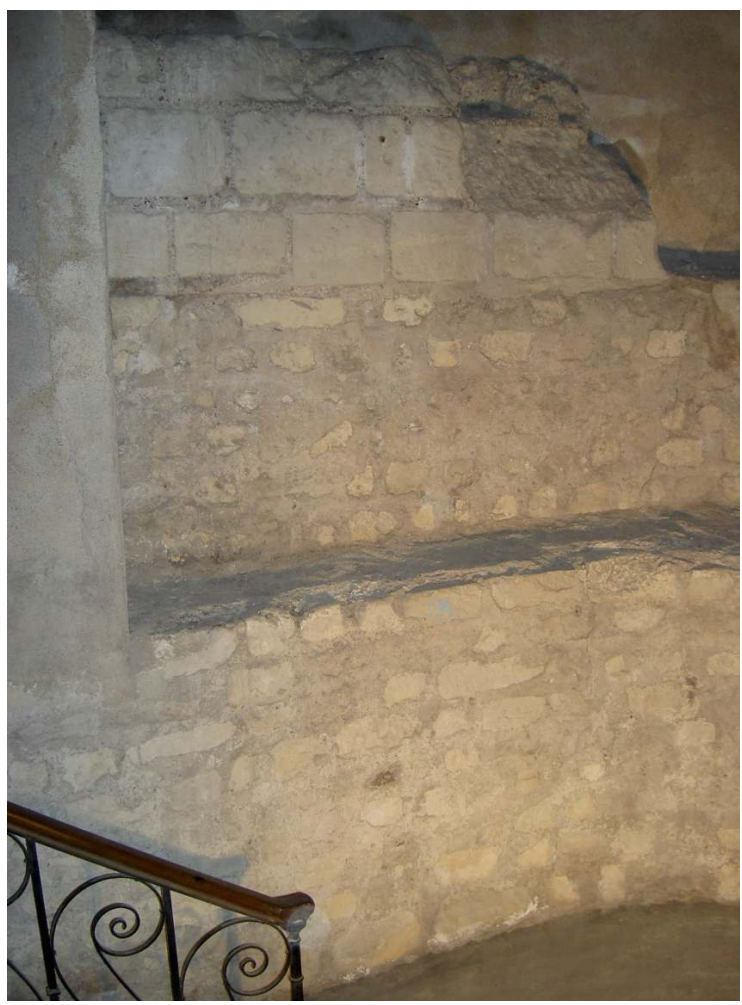

Vestiges de la chapelle axiale, côté nord.

Cl. P. Martin.

Ces rares vestiges ont été relevés, analysés ${ }^{4}$ et mis en perspective avec les observations issues du compte-rendu des fouilles de $1886^{5}$. 


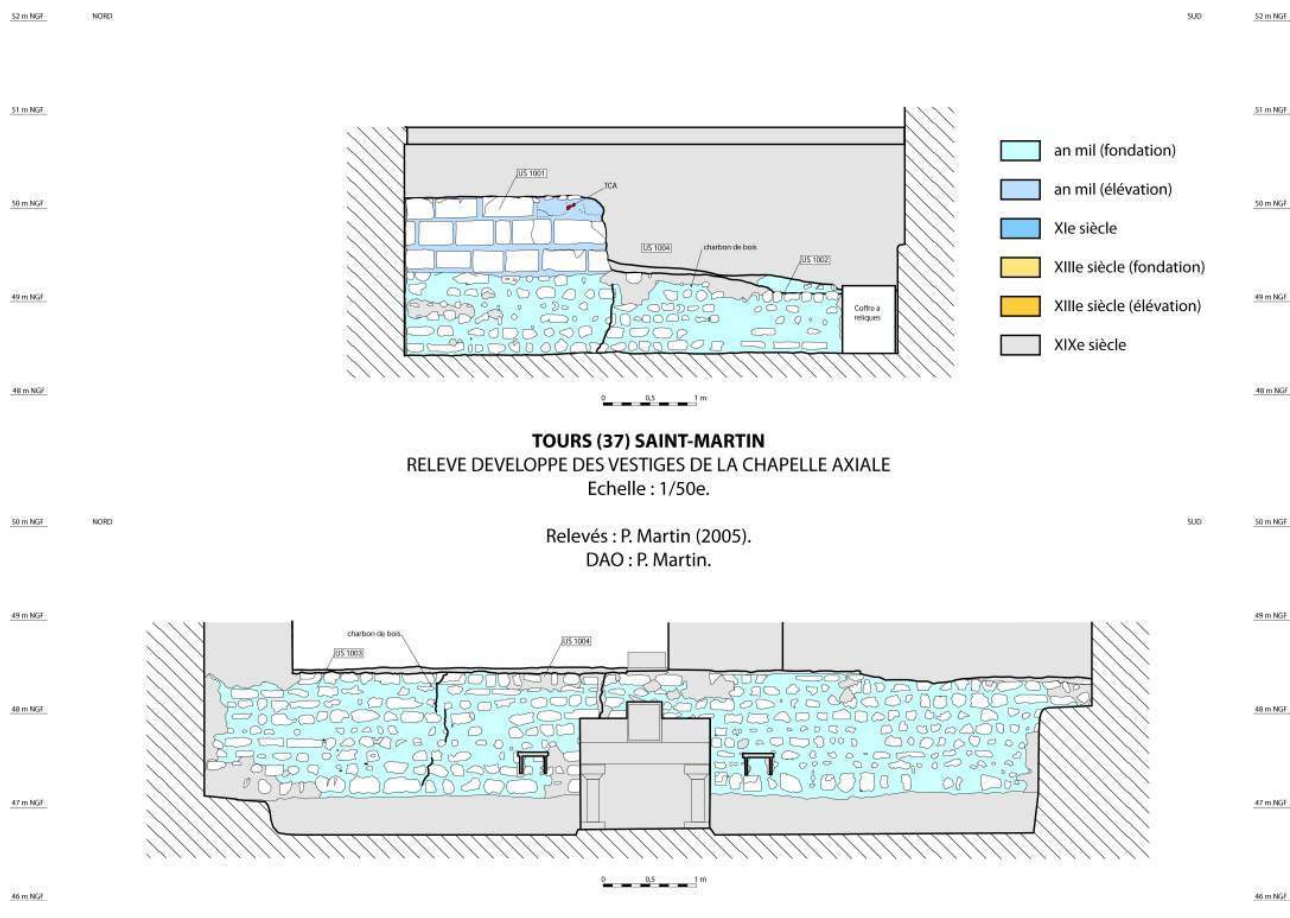

Relevé développé des vestiges de la chapelle axiale. Échelle : 1/50e.

Relevés et DAO : P. Martin.

6 Ainsi a-t-on pu constater la grande qualité du travail de topographie réalisé à la fin du XIX ${ }^{e}$ siècle et prouver l'homogénéité entre fondations et élévations. Ce dernier point avait en effet été contesté, arguant de l'amincissement par paliers des maçonneries. Casimir Chevalier y reconnaissait en partie basse la basilique du $\mathrm{V}^{\mathrm{e}}$ siècle et en partie supérieure le chevet de l'an mil'. Nombre d'archéologues ont suivi cette opinion mais en rejetant l'attribution paléochrétienne pour préférer le $\mathrm{X}^{\mathrm{e}}$ siècle $^{7}$. D'autres ont reconnu deux phases distinctes témoignant de l'évolution du chantier au cours du $\mathrm{XI}^{\mathrm{e}}$ siècle $^{8}$. En souscrivant au contraire à une lecture homogène de l'ensemble des maçonneries, Charles Lelong a tout d'abord associé la construction du chevet à la consécration célébrée en 1096 9. Il est ensuite revenu progressivement sur ses positions pour l'attribuer aux années 1070-1080 ${ }^{10}$, comme les autres grands sanctuaires des routes de Saint-Jacques de Compostelle. Cependant, la reprise du dossier documentaire, l'analyse archéologique des structures encore conservées et la réflexion sur le contexte artistique concourent à réorienter le débat vers une datation haute de la construction.

\section{Les sources relatives au chantier}

Les sources écrites relatives à la reconstruction de Saint-Martin de Tours autour de l'an mil sont principalement constituées par les Histoires de Raoul Glaber et une série de mentions plus ou moins laconiques issues de chroniques, le plus souvent locales et postérieures aux évènements ${ }^{11}$. À la fin du $\mathrm{x}^{\mathrm{e}}$ siècle, un terrible incendie ravagea le bourg de Châteauneuf, la collégiale ainsi que vingt-deux autres églises du quartier ${ }^{12}$. Le corps de saint Martin fut alors transféré dans une chapelle provisoire durant vingt ans. On célébra, au milieu des années 1010, une consécration au cours de laquelle la dépouille du saint 
évêque fut replacée dans l'église. Les financements de ces travaux furent, en toute logique, colossaux et le chantier devait être très avancé vers 1020 car le trésorier de la communauté, Hervé de Buzançais, fut inhumé dans le nouvel édifice.

La translation du corps de saint Martin permet d'aborder la question de la topographie du site. La prestigieuse relique reposait dans l'abside de la basilique paléochrétienne ${ }^{13}$. Le besoin de transférer sa dépouille indique de façon certaine que les travaux ne permettaient plus une continuité du culte autour de la sépulture et que ceux-ci durent avoir une certaine envergure. Les sources précisent en effet que le corps saint fut transféré dans une église de bois située dans le cloître canonial ${ }^{14}$. Elles s'accordent également pour dire que le saint y demeura durant vingt ans, ce qui laisse augurer le développement d'un chantier particulièrement ambitieux. Cette période était suffisamment longue, à condition de maintenir une régularité des financements et la fidélité de grands mécènes, pour voir sortir de terre un grand monument. On peut donc penser que la translation correspond à l'ouverture d'un vaste chantier qui s'est développé à l'est de la basilique du haut Moyen Âge et que l'on prévoyait dès lors un nouvel aménagement monumental pour le tombeau du saint.

9 Le chantier fut financé par l'envoi de fortes sommes d'argent, comme le don de l'impératrice Adélaïde ( $†$ 999) qui y ajouta un morceau du manteau de son fils, Otton II ( $†$ 983), en souvenir du geste de saint Martin aux portes d'Amiens ${ }^{15}$. La reine Adélaïde († 1006), femme d'Hugues Capet († 996), fit également réaliser une chasuble d'or fin et trois chapes tissées d'or ou d'argent ${ }^{16}$. Robert le Pieux $(\dagger 1031)$ participa indirectement, semble-t-il, par un acte en faveur de la collégiale: le second roi capétien confirma la fondation de l'église Notre-Dame de Beaumont et tous ses biens situés dans le voisinage ${ }^{17}$. Peut-être le comte d'Anjou Foulque Nerra (†1040) pourvut-il également à la reconstruction du sanctuaire où ses ancêtres avaient élu sépulture ${ }^{18}$ mais aucun document ne permet de le confirmer.

10 Vers 1001, Hervé de Buzançais fut désigné comme trésorier par Robert le Pieux qui était également abbé laïc de Saint-Martin. En choisissant Hervé, le souverain se tournait vers un homme formé au monastère de Fleury, dont l'abbé Abbon était un proche de la nouvelle dynastie ${ }^{19}$. Si cette nomination reste énigmatique par rapport au déroulement du chantier de Saint-Martin, les sources écrites attribuent unanimement à Hervé la reconstruction de la collégiale ${ }^{20}$. Par son lignage, son rang et son rôle au sein du chapitre, on peut supposer qu'il usa de sa fortune personnelle ${ }^{21}$ pour financer le chantier. Raoul Glaber, qui situe le début des travaux en 1003, précise qu'Hervé fit raser l'ancienne basilique pour la reconstruire de fond en comble, plus vaste et plus haute. Pour ce faire, il indiqua aux maçons où placer les fondations ${ }^{22}$.

11 La consécration solennelle de l'édifice fut sans doute célébrée un 4 juillet, à la fête estivale de saint Martin. On ignore toutefois l'année exacte de l'événement. Les chroniques tourangelles rapportent qu'elle eut lieu du vivant d'Hervée ${ }^{23}$, en 1014 ou $1015^{24}$. Une seule d'entre elles indique la présence de l'archevêque Hugues de Châteaudun qui administra la province jusqu'en $1023^{25}$. Raoul Glaber précise qu'après les travaux, Hervé rassembla plusieurs évêques et abbés pour la consécration et qu'il fit pratiquer la translation de la dépouille de saint Martin dans la nouvelle basilique. Une foule nombreuse des deux sexes et de tous les ordres de la société assista à l'évènement ${ }^{26}$. Raoul Glaber rapporte encore qu'Hervé de Buzançais fut inhumé « au lieu même où reposait le bienheureux Martin »"27. Cependant, Adémar de Chabannes indique seulement qu'il « fut enseveli aux pieds du crucifix ", au milieu de la nef ${ }^{28}$. Un acte de Robert le Pieux fournit également une mention 
complémentaire : Hervé reposait près d'un autel dédié à la Sainte-Croix et à Saint-Pierre situé au milieu de l'église ${ }^{29}$. S'il ne fait donc nul doute qu'Hervé reposait dans la collégiale, probablement entre les septième et neuvième travées de la nef, à proximité de l'entrée du chœur des chanoines ${ }^{30}$, la réutilisation de la sépulture de saint Martin ne semble pas avérée. Seul Raoul Glaber la mentionne, alors que ni ses contemporains ni les sources postérieures n'y font référence. Peut-être le chroniqueur extrapola-t-il ce détail en raison du rôle joué par le trésorier dans la reconstruction de la basilique et le caractère de sainteté qui lui fut reconnu dès sa mort ${ }^{31}$.

Les sources écrites restent ensuite silencieuses et ne mentionnent la collégiale qu'à l'extrême fin $\mathrm{du} \mathrm{xI}{ }^{\mathrm{e}}$ siècle car elle fut la proie d'un incendie puis consacrée lors de la venue d'Urbain II en $1096^{32}$. Deux autres sinistres sont indiqués durant le xII ${ }^{\mathrm{e}}$ siècle : l'un en $1122^{33}$, l'autre en 1157 qui semble d'ailleurs avoir épargné le sanctuaire ${ }^{34}$. Enfin, à partir de 1175, l'édifice fut profondément transformé par la réfection des voûtes de la nef avec un exhaussement du vaisseau central pour le doter d'un éclairage direct ${ }^{35}$. Au début du XIII ${ }^{e}$ siècle, le chevet fut totalement reconstruit selon un parti architectural proche de celui de la cathédrale de Bourges ${ }^{36}$.

\section{Les données archéologiques}

Les textes permettent donc d'affirmer qu'un grand chantier s'ouvrit autour de l'an mil pour reconstruire Saint-Martin de Tours. Les fouilles de 1886 ont également démontré l'absence d'un édifice du haut Moyen Âge à l'emplacement du chevet du XI ${ }^{e}$ siècle tout en mettant au jour ses fondations et la base de son élévation. Par le recoupement des observations anciennes et d'une nouvelle analyse, il est désormais possible de proposer de nouveaux éléments pour appréhender la datation du chevet de la collégiale tourangelle.

\section{Les fondations}

Les fondations du chevet de Saint-Martin de Tours offrent des éléments particulièrement intéressants sur les techniques de construction et l'organisation du chantier. Elles montrent en effet l'adaptation des constructeurs aux contraintes liées à la nature du sous-sol. Les travaux de terrassement ont été considérables. Les maçons assirent sur le substrat sableux (altitude : \pm 45,60 m NGF) des fondations extrêmement puissantes atteignant $3 \mathrm{~m}$ de largeur et s'amincissant progressivement pour soutenir l'élévation, plus ou moins centrée sur ces massifs. La largeur des fondations devait servir à stabiliser la construction sur un terrain assez meuble et sujet aux inondations ${ }^{37}$ de façon à en répartir la charge. Toutefois, on peut s'interroger sur l'absence apparente de radiers formant un grillage de fondation. Cette omission des concepteurs fut sans doute préjudiciable car il n'y avait pas de liaison suffisante entre les charges exercées sous l'hémicycle et sous la couronne de chapelles rayonnantes. Elles-mêmes assuraient un rôle de contrebutement de l'abside. Ainsi Casimir Chevalier a-t-il repéré deux lézardes importantes dans les maçonneries, sur le même axe transversal à l'édifice ${ }^{38}$.

Le problème semble d'ailleurs avoir été récurrent. La fondation de la chapelle axiale fut en effet consolidée par un second massif développé en arc de cercle autour d'elle. Ce renfort était juché sur une forêt de pieux de chêne et un cadre de bois revenant sous la fondation de la chapelle rayonnante sud-est, selon une méthode connue pour les constructions en milieu humide ${ }^{39}$. L'opération implique de nouveaux travaux de 
terrassement très importants car il fallait à nouveau atteindre la base des fondations existantes et travailler en sape. Située à l'extrémité orientale de l'édifice, cette consolidation constituait sans doute pour les constructeurs le point le plus important à stabiliser.

\section{Les élévations}

Les rares vestiges en élévation montrent, à partir de \pm 48,50 m NGF, une construction en moyen appareil à joints rubanés. Le plan présente par ailleurs des chapelles rayonnantes, épaulées par des contreforts assez peu puissants (environ $0,40 \mathrm{~m}$ de profondeur), qui viennent se greffer directement sur les parois intérieures et extérieures du déambulatoire. Les techniques de construction et le désaxement de l'élévation sur les fondations ont longtemps servi d'argument à des datations différentes entre la fondation et l'élévation. Cependant, l'homogénéité des mortiers prouve qu'il s'agit d'une même construction. L'emploi généralisé du moyen appareil peut également être utilisé comme argument chronologique. L'utilisation d'un calcaire relativement tendre permettait de recourir à ce type de mise en œuvre dont les sources du début du XI ${ }^{e}$ siècle ont souligné le caractère de nouveautét ${ }^{40}$. Les parements conservent encore des traces de taille au ciseau, qui renvoient plutôt à des réalisations précoces, de l'époque carolingienne ou du tout début du $\mathrm{XI}^{\mathrm{e}}$ siècle ${ }^{41}$. Le moyen appareil est d'ailleurs à mettre en perspective avec les chronologies régionales. La datation par dendrochronologie du donjon de Loches ${ }^{42} \mathrm{a}$ eu un éclat retentissant sur la datation de bon nombre de monuments prestigieux.

Le plan fournit également quelques indications intéressantes pour aiguiller les hypothèses de restitution de l'édifice. D'après Casimir Chevalier, les piles de l'hémicycle $\mathrm{du} \mathrm{XI}^{\mathrm{e}}$ siècle avaient été chemisées au XIII ${ }^{\mathrm{e}}$ siècle ${ }^{43}$. À la lecture de son plan, cette hypothèse semble toutefois peu probable. Ainsi la présence, à l'origine, de colonnes pour l'hémicycle semble-t-elle relever d'un a priori. Quelques exemples de chevets à déambulatoire précoces présentent en effet des hémicycles dotés de piliers quadrangulaires, comme Notre-Dame de Mehun-sur-Yèvre et San Père de Rodes, de piles composées, comme Sainte-Croix d'Orléans et Saint-Denis de Nogent-le-Rotrou ou encore de supports alternés, comme Saint-Martial de Toulx-Sainte-Croix ou Saint-Étienne de Vignory. 
Nogent-le-Rotrou (28), ancienne église Saint-Denis

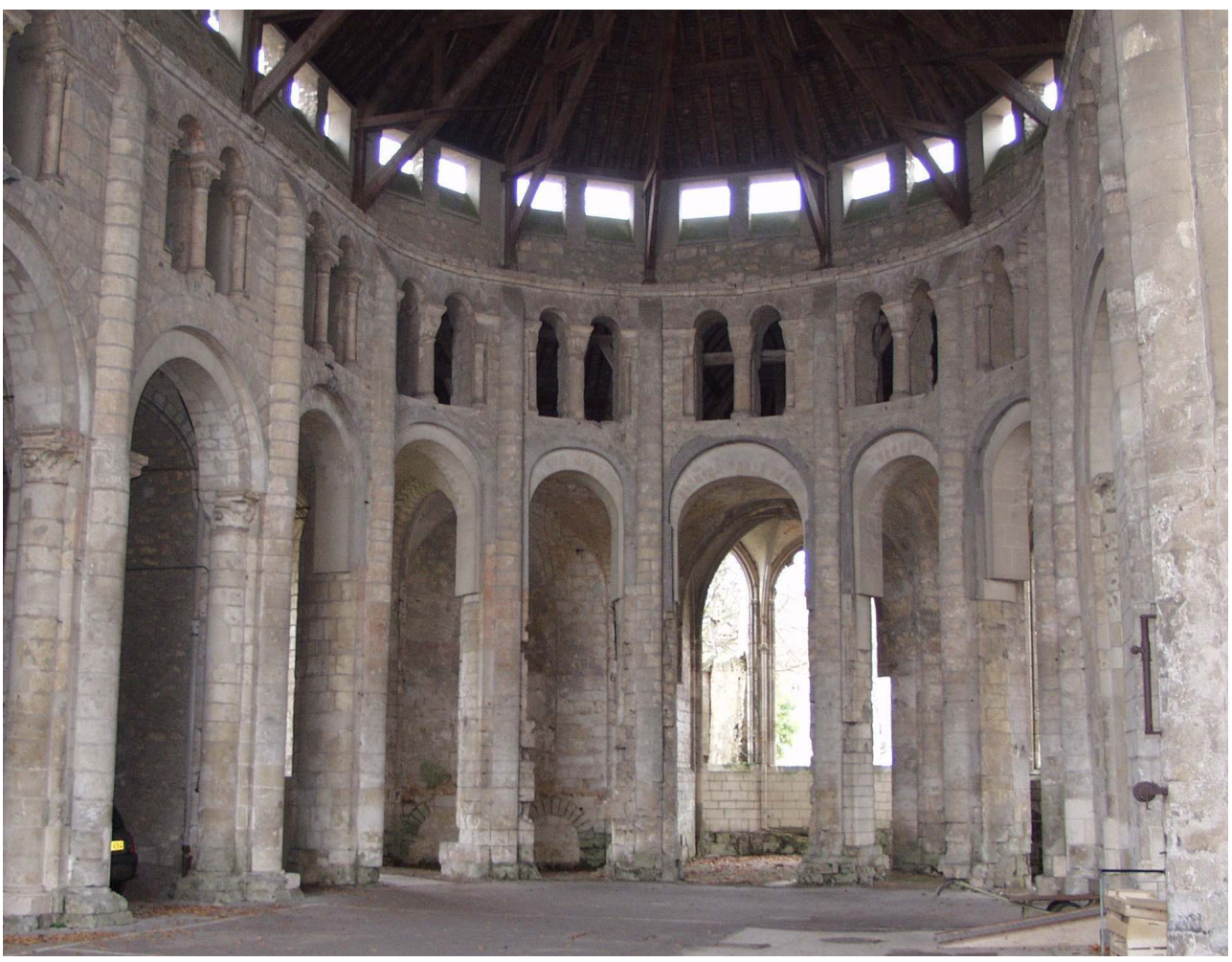

Chevet, élévation intérieure.

Cl. P. Martin. 
Toulx-Sainte-Croix (23), église Sainte-Croix-Saint-Martial.

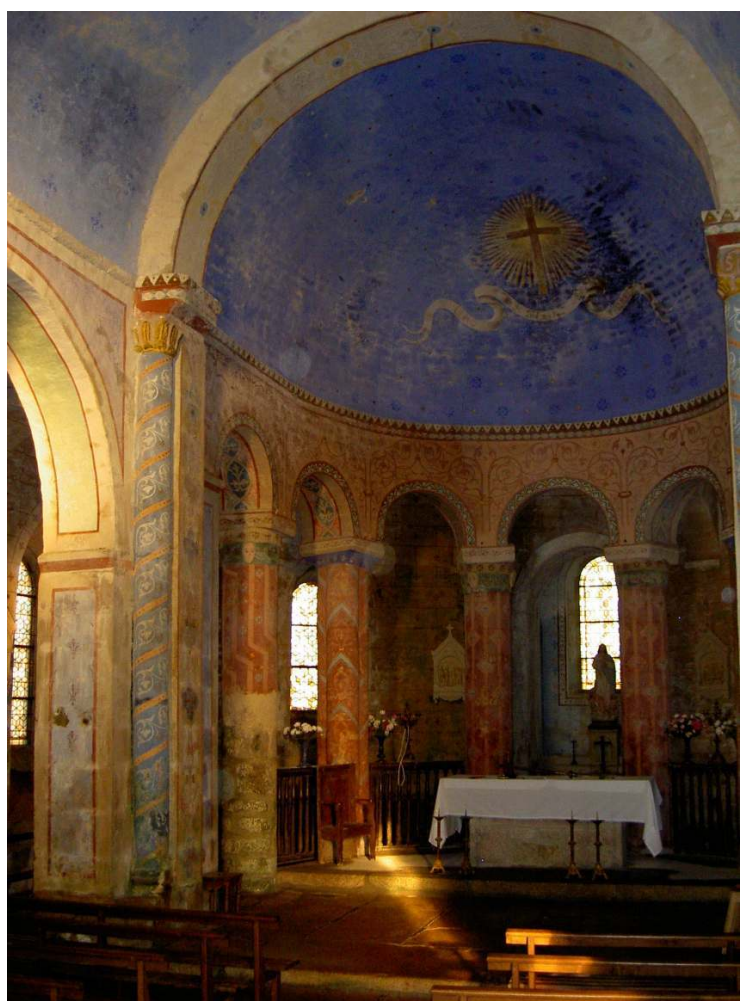

Chevet, élévation intérieure.

Cl. P. Martin

Aussi doit-on considérer que la présence de colonnes dans l'hémicycle de Saint-Martin de Tours, selon une formule très largement répandue, comme à Saint-Savin-sur-Gartempe, ne s'avère être qu'une hypothèse parmi la complexité des formes mises en œuvre dans la première moitié du XI ${ }^{e}$ siècle.

19 Les vestiges de l'élévation du déambulatoire et des chapelles rayonnantes apportent également quelques informations, surtout dans une réflexion planimétrique. D’emblée, on note une grande homogénéité du tracé avec une absence d'articulation entre les absidioles et le couloir annulaire, comme à Saint-Aignan d'Orléans. Cette formule simple, qu'on observe également à Saint-Philibert de Tournus où les chapelles sont quadrangulaires, est enrichie dans des exemples plus tardifs. C'est le cas notamment en Touraine ou dans la vallée du Cher où des colonnes, parfois juchées sur des piédestaux ou multipliées en faisceaux, viennent garnir l'angle formé par les maçonneries et amortissent ainsi la juxtaposition des volumes. En ce sens, le chevet de Saint-Martin de Tours se différencie de ceux des églises dites de pèlerinage, comme Saint-Sernin de Toulouse ou Sainte-Foy de Conques.

En outre, on trouve deux départs de maçonneries quadrangulaires d'environ $0,50 \mathrm{~m}$ de profondeur à l'entrée des chapelles rayonnantes. Elles peuvent être interprétées comme des piédestaux soutenant des colonnes (engagées?) encadrant l'entrée des chapelles, voire rythmant les travées du déambulatoire, comme à Tournus (où les colonnes sont juchées sur un petit soubassement continu). Toutefois, elles pouvaient plus probablement correspondre à des arcatures murales géminées - sans doute situées sous une baie -, comme à Saint-Aignan et à Sainte-Croix d'Orléans, à Nogent-le-Rotrou, à Saint-Savin-sur- 
Gartempe ou à Vignory. Cependant, on ne saurait exclure, comme à Mehun-sur-Yèvre, la présence de simples pilastres montant de fond pour soutenir un voûtement d'arêtes.

\section{Un parti architectural difficile à appréhender}

21 Dégagés il y a plus d'un siècle selon des méthodes aujourd'hui révolues, les vestiges du chevet de Saint-Martin de Tours sont désormais rares et coupés de tout contexte archéologique. Il est donc extrêmement difficile de vouloir conclure de façon catégorique sur un dossier qui reste à traiter à l'échelle de l'édifice. Toutefois, on a vu plus haut que des parallèles pouvaient probablement être esquissés avec des chevets à peu près contemporains.

De ces évocations, il ressort trois hypothèses de restitution pour l'élévation du chevet de Saint-Martin de Tours au début $\mathrm{du} \mathrm{XI}^{\mathrm{e}}$ siècle. La première serait de voir une élévation entièrement voûtée à un seul niveau - les arcades de l'hémicycle -, comme à Vignory ou à Toulx-Sainte-Croix. Dans ce cas, le déambulatoire, lui aussi voûté, pouvait servir de contrebutement au cul-de-four de l'abside. La seconde et la troisième hypothèses tendraient à restituer deux niveaux d'élévation dès l'origine mais selon des dispositions différentes. Dans le premier cas, on pourrait envisager que les arcades de l'hémicycle étaient surmontées par un étage d'éclairage direct mais que l'abside était charpentée, comme à Bray-sur-Seine. Dans le second cas, on peut proposer des tribunes surmontant les arcades de l'hémicycle et assurant ainsi le contrebutement d'un cul-de-four sur l'abside. C'est ce que pourrait suggérer l'exemple de Saint-Denis de Nogent-le-Rotrou.

En l'absence de certitude, on privilégiera l'hypothèse d'une solution voûtée tant les premiers chevets à déambulatoire semblent avoir plus largement développé ce type de formule, comme à Vignory ou à Toulx-Sainte-Croix ${ }^{44}$. Le couloir annulaire enveloppant l'abside permettait, certes, de circuler facilement dans l'édifice tout en desservant des espaces secondaires et de nombreux autels mais il jouait également un rôle structurel pour mettre en place une élévation pyramidale des volumes extérieurs. L'ambition des constructeurs fut probablement l'objet de controverses architecturales. De nombreuses absides montrent en effet des repentirs ou des reconstructions de l'étage d'éclairage direct, à partir de la seconde moitié du XI ${ }^{e}$ siècle - comme à Mehun-sur-Yèvre ou à SaintSavin -, c'est-à-dire à une époque où les voûtements étaient déjà plus largement répandus et surtout mieux maîtrisés. Quoi qu'il en soit, les solutions à envisager pour la restitution de l'élévation du chevet de Saint-Martin de Tours semblent assez différentes des formules voûtées à trois niveaux d'élévation qui furent développées à partir du milieu du XI $\mathrm{XI}^{\mathrm{e}}$ siècle à Saint-Sernin de Toulouse ${ }^{45}$ ou à Sainte-Foy de Conques ${ }^{46}$.

Malgré les incertitudes documentaires et les lacunes archéologiques, on constate qu'un faisceau d'éléments conduit à replacer le chevet de Saint-Martin de Tours parmi les grands chantiers de l'an mil. Après un incendie qui entraîna la translation des reliques du saint pour vingt ans, la collégiale fut rebâtie sous l'action du trésorier Hervé de Buzançais et consacrée au milieu des années 1015. Hervé fut inhumé dans la nef de l'église vers 1020, ce qui laisse présager un avancement rapide des travaux.

Le parti architectural du nouveau chevet ne peut être abordé qu'avec beaucoup de réserve. Toutefois, il est désormais acquis que les puissantes fondations, destinées à asseoir la construction sur un substrat sableux et sujet aux inondations, supportaient une élévation en moyen appareil. Probablement rythmé par des arcatures murales, le déambulatoire desservait cinq chapelles rayonnantes régulièrement contrefortées. On 
ignore néanmoins le type d'organisation de l'hémicycle mais les comparaisons proposées ouvrent un champ de restitution beaucoup plus large que celui envisagé traditionnellement. Ainsi l'hypothèse d'un rond-point de colonnes ou d'une élévation à trois niveaux constitue les derniers feux de la théorie des églises de pèlerinage. Cette proposition ne semble pas, en effet, refléter la variété des formes mises en œuvre à la même époque, a fortiori dans la France du centre, territoire riche en expériences précoces de chevets à déambulatoire et où l'entourage royal paraît avoir tenu un rôle prépondérant ${ }^{47}$.

Le recours à l'archéométrie semble conforter l'hypothèse d'une datation du chevet de Saint-Martin vers l'an mil. Des de charbons de bois ont été prélevés dans le mortier de la fondation de la chapelle axiale. Une datation radiocarbone sur l'un d'entre eux a fourni un âge calibré de 881-989 AD. Si l'activité radiocarbone couvre ainsi l'ensemble du $\mathrm{X}^{\mathrm{e}}$ siècle, elle semble bien exclure le plein $\mathrm{XI}^{e}$ siècle ${ }^{48}$. Toutefois, seule une multiplication des analyses permettra de confirmer cette tendance. Le dernier argument à souligner réside dans la reconstruction rapide du chevet dès le début du XIII ${ }^{e}$ siècle. Si l'œuvre d'Hervé correspondait déjà à une modernisation de l'édifice du haut Moyen Âge, sa réédification totale après 1200 indique probablement sa vétusté. On peut tenter de l'expliquer par des problèmes techniques ou structurels, notamment des fondations, mais également par le caractère désuet et obscur d'une construction mal éclairée - car totalement voûtée. Ainsi doit-on remettre en cause une nouvelle fois la chronologie de ce grand monument qui devra faire l'objet, à l'avenir, d'une véritable étude monographique.

\section{NOTES}

1. Marie-Laure Crosnier-Leconte, Victor Laloux (1850-1937). L'architecte de la Gare d'Orsay, Paris, RMN, 1987 («Les dossiers du Musée d'Orsay », 9).

2. Émile Mâle, L'art religieux du XII ${ }^{e}$ siècle en France. Étude sur les origines de l'iconographie du Moyen Âge, Paris, Armand Collin, 1922, p. 297-300.

3. Sur ces grands monuments, voir en dernier lieu Henri Pradalier, « Saint-Sernin de Toulouse au Moyen Âge ", Congrès archéologique de France, Toulouse et Comminges, 1996, p. 254-301. Quitterie Cazes, Daniel Cazes, Saint-Sernin de Toulouse. De saint Saturnin au chef-d'œuvre de l'art roman, Graulhet, Odyssée, 2008. Éliane Vergnolle, Henri Pradalier, Nelly Pousthoumis-Dalle, «Conques, Sainte-Foy", Congrès archéologique de France, Aveyron, 2009, p. 71-160. Claude Andrault-Schmitt (éd.), Saint-Martial de Limoges. Ambition politique et production culturelle ( $\mathrm{X}^{e}$-XIII ${ }^{e}$ siècles), Actes du colloque de Poitiers-Limoges, 26-28 mai 2005, Limoges, PULim, 2006. Isidro Gonzalo Bango Torviso, "Arquitectura románica en Galicia. Desde los orígenes hasta 1168 ", dans José Carlos Valle Pérez, Jorge Rodrigues (éd.), Románico en Galicia y Portugal: A arte romanica em Portugal e Galiza, La Coruña, Fundación Pedro Barrié de la Maza, Lisbon, Fundação Calouste Gulbenkian, 2001, p. 12-28. Sur les églises de pèlerinage, voir Beat Brenk, «Les églises de pèlerinage et le concept de prétention ", dans Nicolas Bock, Peter Kurmann, Serano Romano, Jean-Michel Spieser (éd.), Art, cérémonial et liturgie au Moyen Âge, actes du colloque du troisième cycle romand de lettres, Lausanne-Fribourg, 24-25 mars, 14-15 avril, mai 12-13 2000, Rome, Viela, 2002 ("Études lausannoises d'histoire de l'art », 1), p. 125-139. 
4. Des vestiges du bras sud du transept ont également été découverts et partiellement conservés. Pour plus de détails, voir Pierre Martin, Les premiers chevets à déambulatoire et chapelles rayonnantes de la Loire moyenne (Xe-XIe siècles). Saint-Aignan d'Orléans, Saint-Martin de Tours, Notre-Dame de Mehun-sur-Yèvre, La Madeleine de Châteaudun, thèse de doctorat, Andrault-Schmitt (C.) (dir.), 4 vol., université de Poitiers - CéSCM, décembre 2010, vol. 1, p. 95-150.

5. Casimir Chevalier, Les Fouilles de Saint-Martin de Tours. Recherches sur les six basiliques successives élevées autour du tombeau de saint Martin, Tours, L. Péricat, 1888.

6. Sur les arguments de Chevalier et ses répercutions sur le vocabulaire architectural, voir Pierre Martin, "Autour du chœur, le déambulatoire. Terminologie et historiographie ", dans Claude Arrignon, Marie-Hélène Debiès, Claudio Galderisi, Éric Palazzo (éd.), Cinquante années d'études médiévales. À la confluence de nos disciplines, actes du colloque organisé à l'occasion du cinquantenaire du CéSCM, Poitiers, 1er-4 septembre 2003, Turnhout, Brépols, 2005 («Culture et société médiévales »), p. 241-250.

7. Robert de Lasteyrie, L'Église Saint-Martin de Tours, étude critique sur l'histoire et la forme de ce monument du $V^{e}$ au XI ${ }^{e}$ siècle, Paris, C. Klincksieck, 1891, p. 12-13. Gabriel Plat, L'Architecture religieuse en Touraine des origines au XII siècle. L'art de bâtir des Romains à l'an 1100, Paris, 1939 («Mémoires de la Société archéologique de Touraine», 7), p. 40, 58-59, 68. Carl K. Hersey, "The Church of Saint-Martin at Tours (903-1150)», The Art Bulletin, 25, 1, mars 1943, p. 38.

8. Frédéric Lesueur, «Saint-Martin de Tours et les origines de l'art roman ", Bulletin monumental, 107, 1949, p. 48-58. Hans Sedlmayr, "Saint-Martin de Tours im XI. Jahrhundert ", Bayerische Akademie der Wissenschaften, 69, 1970, p. 9-40. Pour le reste de la bibliographie, voir Pierre Martin, "Premières expériences de chevets à déambulatoire et chapelles rayonnantes de la Loire moyenne. État de la question ", Cahiers de Saint-Michel de Cuxa, 32, 2001, p. 184-186.

9. Charles Lelong, «La date du déambulatoire de Saint-Martin de Tours », Bulletin monumental, 131, 1973, p. 308.

10. Charles Lelong, La Basilique Saint-Martin de Tours, Chambray-lès-Tours, CLD, 1986, p. 78.

11. Mathieu Arnoux (éd.), Raoul Glaber, Histoires, Turnhout, Brépols, 1996. Yves Chauvin, Georges Pon (éd.), Adémar de Chabannes, Chronique, Turnhout, Brépols, 2003. André Salmon (éd.), Recueil des chroniques de Touraine, Tours, SAT, 1854.

12. La date exacte de cet incendie reste difficile à préciser, entre 994 et 997 selon les sources. Cette question a fait débat mais s'avère finalement secondaire. On notera toutefois que Raoul Glaber ne fait pas mention de ce sinistre.

13. Christian Sapin, "Saint-Martin de Tours, $\mathrm{V}^{\mathrm{e}}-\mathrm{X}^{\mathrm{e}}$ siècles ", dans Henri Galinié (dir.), Tours antique et médiéval. Lieux de vie, temps de la ville. Quarante ans d'archéologie urbaine, catalogue de l'exposition du Château de Tours, 14 octobre 2006-18 mars 2007, Tours, FERACF, p. 255.

14. Recueil des chroniques de Touraine, op. cit., p. 229, 116, 301. Guibert de Gembloux, « De cultu S. Martini apud Turonenses extr. Sec. XII, Epistolae quatuor », Annalecta bollandiana. Revue critique d'hagiographie, 3, 1884, p. 227.

15. Odilonis Cluniacensis abbas, Epitaphium Adelheide Imperatricis, dans Jacques-Paul Migne (éd.), Patrologia latina, 142, Paris, 1853, col. 979. Sean Gilsdorf (éd.), Queenship And Sanctity: The Lives Of Mathilda And The Epitaph of Adelheid, Washington, CUAP, 2004, p. 139-140.

16. Robert-Henri Bautier, Gilette Labory (éd.), Helgaud de Fleury, Vie de Robert le Pieux. Epitoma vitae regis Rotberti Pii, Paris, CNRS, 1965 (« Sources d'Histoire médiévale », 1), p. 81-85.

17. William Mendel Newman (éd.), Catalogue des actes de Robert le Pieux, Paris, Librairie du Recueil Sirey, 1937, n³0, 92.

18. Louis Halphen, René Poupardin (éd.), Chroniques des comtes d'Anjou et des seigneurs d'Amboise, Paris, Picard, 1913, p. 16, n. 85, n. 233 et p. 34, 37.

19. Helgaud de Fleury..., op. cit., p. 84, n. 1. Abbon avait par ailleurs été très proche d'Hervé quand il étudiait à Fleury. Guy-Marie Oury, "L'idéal monastique dans la vie canoniale. Le bienheureux Hervé de Tours († 1022)», Revue Mabillon, 207, 1962, p. 5-6. 
20. Recueil des chroniques de Touraine, op. cit., p. 116, 187, 229, 301. Raoul Glaber..., op. cit., p. 165-169. Adémar de Chabannes, op. cit., p. 265, 283-284.

21. Guy-Marie Oury, «L'idéal... », op. cit., p. 3, n. 2.

22. Raoul Glaber..., op. cit., p. 165.

23. Deux dates sont données pour la mort d'Hervé de Buzançais : 1012 et 1022. On notera toutefois que la première paraît douteuse. Recueil des chroniques de Touraine, op. cit., p. 119. La première mention du successeur d'Hervé à la charge de trésorier, Sulpice d'Amboise, remonte à 1023. Catalogue des actes de Robert le Pieux, op. cit., $\mathrm{n}^{\circ} 92$.

24. Recueil des chroniques de Touraine, op. cit., p. 30, 51, 119, 229. Guibert de Gembloux, op. cit., p. 227.

25. Recueil des chroniques de Touraine, op. cit., p. 229. La dernière édition de la Chronique d'Adémar de Chabannes place pourtant, d'après des recoupements, la consécration de Saint-Martin vers 1026. Adémar de Chabannes, op. cit., p. 265.

26. Raoul Glaber..., op. cit., p. 167-169.

27. Ibid., op. cit., p. 170-171.

28. Sur l'interprétation, voir Adémar de Chabannes, op. cit., p. 283-284, n. 568.

29. Catalogue des actes de Robert le Pieux, op. cit., $\mathrm{n}^{\circ} 92$.

30. Charles Lelong, La basilique..., op. cit., p. 110.

31. Catalogue des actes de Robert le Pieux, op. cit., $\mathrm{n}^{\circ}$ 92. Adémar de Chabannes, op. cit., p. 283-284.

32. Recueil des chroniques de Touraine, op. cit., p. 59, 129. Louis Halphen, Le Comte d'Anjou au XI ${ }^{e}$ siècle, Paris, Picard, 1906, p. 67. Chroniques des comtes d'Anjou..., op. cit., p. 381. Eugène Mabille, « Notice sur les divisions territoriales et la topographie de l'ancienne province de Touraine ", Bibliothèque de l'École des chartes, 1864-1865, p. 112, n. 2. Edmond Martène, «Histoire de l'abbaye de Marmoutier ", Mémoires de la Société archéologique de Touraine, 24, 1874, p. 69.

33. Recueil des chroniques de Touraine, op. cit., p. 62, 132, 302.

34. Ibid., p. 302.

35. Ibid., p. 302. Tours, Bibliothèque municipale, ms n 1 294-1 295, 1, p. 234.

36. Charles Lelong, La basilique..., op. cit., p. 79-90, 91-98. Charles Lelong, «Indre-et-Loire. Remarques sur le chevet gothique de Saint-Martin de Tours (Actualité) », Bulletin monumental, 149, 1991, p. 229-230. Claude Andrault-Schmitt, «La cathédrale de Tours. Le chevet du XIII siècle », Congrès archéologique de France. Touraine, 1997, p. 295.

37. L'inondation survenue en cours de fouille le 13 août 1886 atteignit la cote 46,95 m NGF. Casimir Chevalier, Les fouilles..., op. cit., pl. 7.

38. Ce type de désordre de fondation est fréquent et est généralement causé par des infiltrations désagrégeant les mortiers ou par une variation de la nature du sol. Yves-Marie Froidevaux, Techniques de l'architecture ancienne. Construction et restauration, $4^{\mathrm{e}}$ éd., Sprimont, Mardaga, 1986, p. 155.

39. Gabriel Plat, L'architecture..., op. cit., p. 46-47. Prélevés en 1886, ces pieux sont conservés dans une réserve de la basilique moderne. Toutefois, l'absence d'aubier n'a pas permis de recourir à des datations dendrochronologiques.

40. Éliane Vergnolle, «La pierre de taille dans l'architecture religieuse de la première moitié du $\mathrm{XI}^{\mathrm{e}}$ siècle ", Bulletin monumental, 154, 1996, p. 229-234.

41. Gabriel Plat, L'architecture..., op. cit., p. 18-48. Christian Sapin, «Modes de construction et appareils de pierre carolingiens : quel héritage pour l'époque romane ? Problèmes historiques et archéologiques ", Cahiers de Saint-Michel de Cuxa, 37, 2006, p. 77-89.

42. Jean Mesqui, «La tour maîtresse du donjon de Loches ", Bulletin monumental, 156, 1998, p. 100-101.

43. Casimir Chevalier, Les fouilles..., op. cit., p. 124.

44. Pierre Martin, "L'église Saint-Martial à Toulx-Sainte-Croix », dans Claude Andrault-Schmitt (éd.), Saint-Martial de Limoges..., op. cit., p. 287-298. 
45. Henri Pradalier, « Saint-Sernin ... », op. cit. Quitterie Cazes, Daniel Cazes, Saint-Sernin..., op. cit., p. 61-72. Éliane Vergnolle et al., « Conques, ... », op. cit.

46. Henri Pradalier, «Saint-Sernin ... », op. cit. Quitterie Cazes, "L'abbatiale de Conques, genèse d'un modèle architectural roman », Cahiers de Saint-Michel de Cuxa, 37, 2006, p. 114, n. 24. Éliane Vergnolle et al., «Conques, ... », op. cit., p. 117-122.

47. Pierre Martin, Les Premiers chevets..., op. cit., vol. 1, p. 299-303, 307-313. Pierre Martin, « SainteCroix d'Orléans. Le chevet du xie siècle", dans Pascal Liévaux (éd.), La Cathédrale Sainte-Croix d'Orléans, actes de la journée d'études du 7 octobre 2011, Orléans, 2012 (à paraître).

48. Les dates les plus probables sont $944,920,898,785$. Pierre Martin, Les Premiers chevets..., op. cit., vol. 4, p. 47-48.

\section{RÉSUMÉS}

Embellie et partiellement reconstruite aux XII ${ }^{\mathrm{e}}$ et XIII ${ }^{\mathrm{e}}$ siècles, la collégiale Saint-Martin de Tours fut détruite à la Révolution. C'est préalablement à une réédification à la fin du XIX ${ }^{\mathrm{e}}$ siècle que le chevet $\mathrm{du} \mathrm{XI}{ }^{\mathrm{e}}$ siècle et le bras sud du transept furent partiellement mis au jour. Les découvertes effectuées en 1886 et les observations faites dans les soubassements de la basilique actuelle permettent de penser que les vestiges du chevet à déambulatoire et chapelles rayonnantes pourraient bien appartenir à la campagne de travaux de l'an mil. Les sources écrites fournissent en effet de nombreux renseignements sur le chantier de reconstruction mené par le trésorier Hervé de Buzançais et sur la consécration qui fut célébrée au milieu des années 1010. Toutefois, la question de la datation absolue de ce célèbre chevet tourangeau a été maintes fois débattue et sujette à polémiques et controverses. Alors que depuis les années 1980-1990, il avait été admis que cette partie de l'édifice remontait à la seconde moitié $\mathrm{du} \mathrm{XI}^{\mathrm{e}}$ siècle, un réexamen des données archéologiques et des contextes historique et artistique conduit à envisager une chronologie haute de cette œuvre.

After being embellished and partially rebuilt in the 12th and 13th centuries, the collegiate church of Saint-Martin de Tours was destroyed during the Revolution. By the time the church was rebuilt in the late 19th century, both the 11th century apse and south transept had been partially discovered. Observations made in 1886 in the foundations of the current basilica suggest that the ambulatory apse with radiating chapels may belong to a rebuilding dating back around year 1000 . Primary sources provide much information about the works led by treasurer Hervé de Buzançais and about a consecration celebrated in the mid 1010s.

However, the question of the absolute dating of the famous apse of Saint-Martin has been debated and controversed for a long time. Since the 1980s, it has been assumed that the apse was dating back the second half of the 11th century. But a new review of archaeological data and historical and artistic backgrounds favour a building's earlier chronology.

Die Stiftskirche Saint-Martin de Tours wurde im Laufe der 12. und 13. Jahrhundert beschönigt und zum Teil neu aufgebaut. Sie wurde während der französischen Revolution zerstört. Beim Wiederaufbau der Kirche am Ende des 19. Jahrhunderts wurden der Chorhaupt aus dem 11. Jahrhundert und der südliche Querschiffarm ausgegraben. Die Entdeckungen im Jahr 1886 und die Erforschung des Fundamentes der gegenwärtigen Basilika weisen darauf hin, dass die Reste des Chorhauptes mit Chorumgang und Kapellenkranz zu den Umbau der Kirche im Jahr 1000 
gehören könnten. Die schriftlichen Quellen erteilen nämlich wichtige Auskünfte über den Wiederaufbau, welcher von dem Schatzmeister Hervé de Buzançais geleitete wurde. Diese Quellen berichten ebenfalls über die Weihung, die in der Mitte der 1010er Jahren stattfand.

Über die Datierung des berühmten Chorhauptes von Tours wurde jedoch viel debattiert. Seit 1980-1990 wird zwar damit einverstanden, dass dieser Bereich der Kirche im zweiten Teil des 11. Jahrhunderts errichtet wurde. Eine erneute Prüfung der archäologischen Angaben und des historischen und kulturellen Kontextes weisen jedoch auf eine frühere Datierung hin.

\section{AUTEUR}

\section{PIERRE MARTIN}

Né en 1975, Pierre Martin est titulaire d'un doctorat en histoire de l'art obtenu en 2010 sous la direction de Claude Andrauld-Schmitt, centre d'Études supérieures de la civilisation médiévale, université de Poitiers : Les premiers chevets à déambulatoire et chapelles rayonnantes de la Loire moyenne ( $\mathrm{X}^{e}-\mathrm{XI} I^{e}$ siècles). Saint-Aignan d'Orléans, Saint-Martin de Tours, Notre-Dame de Mehun-sur-Yèvre, la Madeleine de Châteaudun (consultable en ligne http://tel.archives-ouvertes.fr/tel-00581583/fr/). Il est chercheur associé à l'UMR 6223, centre d'Études supérieures de la civilisation médiévale. Il est actuellement archéologue responsable d'opérations (Archeodunum, SAS, 500 rue Juliette Récamier 69970 Chaponnay).

pierremartin1975@free.fr 